\title{
Ecdyonurus rothschildi Navás, 1929 : description de la larve (Ephemeroptera, Heptageniidae)
}

\author{
T. Soldán 1 \\ J. Gagneur ${ }^{2}$
}

Mots clés : Ephemeroptera, Heptageniidae, Taxonomie larvaire, Algérie.

La larve au dernier stade d'Ecdyonurus rothschildi Navás est figurée et décrite comparativement à celles d'E. aurantiacus (Burmeister) et d' E. dispar (Curtis).

Ecdyonurus rothschildi Navás, 1929 : a description of the larva (Ephemeroptera, Heptageniidae)

Keywords : Ephemeroptera, Heptageniidae, Larval taxonomy, Algeria.

The larva of the last instar of Ecdyonurus rothschildi Navas is figured and described comparatively to those of E. aurantiacus (Burmeister) and of E. dispar (Curtis).

En 1929 Navás a décrit d'Afrique du Nord l'imago - d'Ecdyonurus rothschildi. Le nom de cette espèce, tombé ensuite dans l'oubli, est cité pour la deuxième fois par Puthz (1978).

Thomas et Dakki (1979) ont redécrit des imagos provenant de la localité typique (Biskra, Algérie) et du Moyen Atlas marocain - mais les larves sont restées inconnues.

L'examen de notre matériel (larves capturées en Algérie entre 1981 et 1984 ) rend possible leur description. Les larves au dernier stade d' E. rothschildi sont comparées ici à celles d'E. aurantiacus et d' $E$. dispar (matériel du Sud-Ouest de la France et de Tchécoslovaquie). En effet, cette espèce, par ses caractères imaginaux, appartient au groupe $E$. aurantiacus: voir Thomas et Dakki (l.c.). Descriptions de référence des larves d'E. aurantiacus \{s.n. pazsiczkyi Pongrácz) et d' E. dispar: Thomas (1968).

1. Institute of Entomology, Czechoslovak Academy of Sciences, Na sádkách 702, CS-370 05 Ceské Budejovice, Czechoslovakia.

2. B.P. 465, Centre Universitaire, Tlemcen, Algérie.

\section{1. - Taille et coloration}

La taille des larves est maximale au début de la période de vol ; longueur du corps : 12,5-15,4 mm (larves \&) et 10,5-13,2 mm (larves or) en avril et mai, $9,0-11,5$ (larves 9 ) et $8,5-10,5 \mathrm{~mm}$ (larves $\sigma$ ) au début d'octobre. Cerques et paracerque à peu près de la même longueur que le corps.

Larves foncées, brunes ou brun foncé avec des taches dorsales jaunâtres, assez contrastées et nombreuses sur la tête, le thorax et l'abdomen; pronotum avec une tache large au milieu, segments abdominaux avec deux paires de taches, dont les plus internes sont en forme de " $\mathrm{V}$ *. Ces taches sont plus intenses que chez $E$. dispar. Les larves provenant des environs de Tlemcen sont beaucoup plus foncées dorsalement que les larves de la Chiffa, très claires, jaunâtres (voir Lestage, 1925 s.n. Ecdyonurus sp., espèce jamais décrite). Sur les larves au dernier stade, les tergites abdominaux portent une paire de bandes étroites, obliques allant du brun foncé au noirâtre comme chez $E$. aurantiacus. 


\section{2. - Tête et pronotum}

Tête à peine moins large que le pronotum, légèrement trapézoïdale (fig. 1) semblable à celle de la larve d'E. dispar. Les bords postéro-latéraux sont convexes ou tout au plus rectilignes (rectilignes ou concaves chez E. dispar: fig. 2). Le rapport largeur/longueur de la tête a une valeur moyenne de 1,48 (extrêmes observés : 1,42-1,55) comme chez $E$. dispar. Ce rapport est d'1,70 (1,62-1,80) chez $E$. aurantiacus.

Pronotum (fig. 3) proéminent vers les bords et arrondi, avec expansions paranotales et denticule des angles antérieurs comme chez $E$. dispar. Cette dent est absente ou très réduite chez quelques individus. Chez la larve d'E. aurantiacus, elle est plus longue et plus pointue. La largeur maximale du demi-pronotum est inférieure à sa longueur.

Le rapport largeur/longueur est de 1,15.1,25 comme chez $E$. dispar (1,35-1,45 chez E. aurantiacus). Expansions paranotales étroites à portion apicale en pointe mousse, reliées au mésothorax (expansions libres chez $E$. aurantiacus et $E$. dispar). La longueur des expansions est contenue environ 4 fois $(3,70-4,55)$ dans la longueur totale du pronotum. Par ce seul caractère, les larves ne peuvent être séparées de celles d' E. dispar et d'E. aurantiacus. Thomas (1968) indique des valeurs respectives de 3,46-4,00 et de $4,46-5,83$ pour ces dernières espèces.

\section{3. - Pièces buccales}

Labre (fig. 4) assez étroit, semblable à celui d'E. aurantiacus, avec les côtés arrondis et très légèrement concaves vers l'arrière. Surface dorsale du labre avec deux rangées obliques d'épines plus fortes et plus nombreuses (8-10) que chez E. aurantiacus et $E$. dispar. Le rapport de sa largeur totale à la longueur de son insertion est de 2,30-2,75 : d'après Thomas (1968), il est de 2,22 à 2,86 chez E. aurantiacus et de 1,69 à 2,04 chez E. dispar. Prostheca com. posée de 15-19 fortes soies, plus courtes d'un tiers que les canines intérieures. Prostheca des larves d' $E$. aurantiacus (fig. 6) et E. dispar composée de 5-8 soies. Lacinia pourvue de 15-17 peignes comme chez $E$. aurantiacus (17-20 peignes chez $E$. dispar). La longueur du troisième article du palpe maxillaire est contenue 6 fois, et parfois même 7, dans la longueur du deuxième article comme chez $E$. aurantiacus (troisième article plus court chez E. dispar). Lobe externe de l'hypopharynx concave vers l'arrière avec une pilosité descendant loin vers le bas, le long de sa marge extérieure (fig. 8). Le contour apical des glosses (fig. 7) est à peu près rectiligne et presque perpendiculaire au plan de symétrie comme chez les larves d'E. aurantiacus. Chez E. dispar, au contraire, il a une forme en " $\mathrm{V}$ " (voir Thomas 1968: fig. 7).

\section{4. - Pattes}

Tarses assombris sur toute leur longueur, surtout dans leur partie terminale. Griffes avec 3 grandes dents (quelques larves avec deux dents). En général 5 pattes sur 6 portent chacune trois dents sous la griffe. Nous n'avons pas observé ( 50 griffes examinées) de griffes portant 4 dents, comme chez environ $15 \%$ des larves d' $E$. dispar étudiées par Tho mas (1968).

Coloration dorsale des fémurs I à III : fig. 16. Toute la surface dorsale des fémurs est couverte d'épines plus ou moins nettement arrondies au sommet (fig. 12) comme chez E. aurantiacus. Les épines des larves d' $E$. dispar sont plus longues et pointues au sommet (fig. 13).

\section{5. - Branchies et segments abdominaux}

Lamelle de la première branchie (fig. 8) nettement rétrécie vers l'apex, étroite, $4-5$ fois plus longue que large, comme chez E. aurantiacus. Cette lamelle est 2,5-3 fois plus longue que large chez $E$. dispar. Lamelles de la seconde à la $6^{\circ}$ branchie ovales ( $f$ ig. 10), presque symétriques, leur moitié supérieure colorée en brun violet. $7^{\circ}$ branchie légèrement asymétrique, sans touffe de filaments trachéens (fig. 11).

Epines latéro-abdominales (fig. 15) assez courtes, parallèles à l'axe longitudinal du corps et même très légèrement courbées vers lui, atteignant sur les segments moyens $1 / 5$ ou 1/6 de la longueur totale de ces derniers. Bord postérieur des tergites couvert d'épines aiguës bien développées, de trois classes de taille différentes (fig. 14). Chez E. dispar et E. aurantiacus, les différences de longueur de ces épines sont moins marquées. 

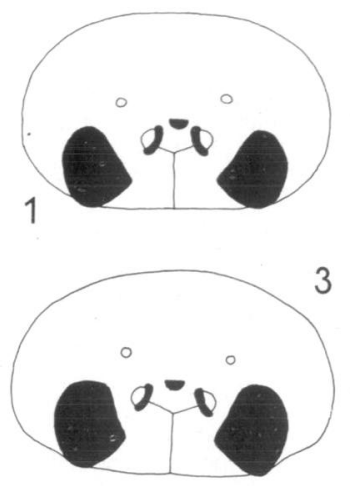

$2 \quad 1.5 \mathrm{~mm}$
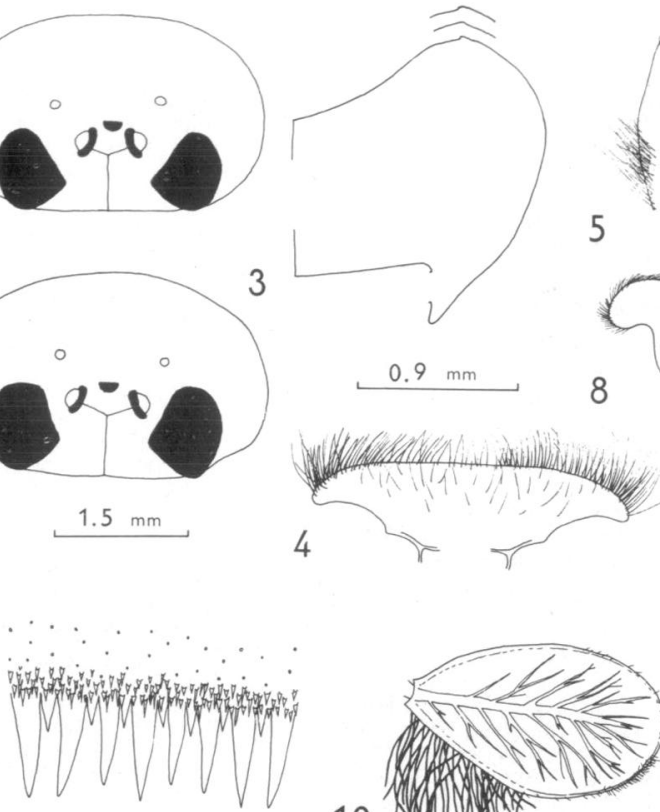

14
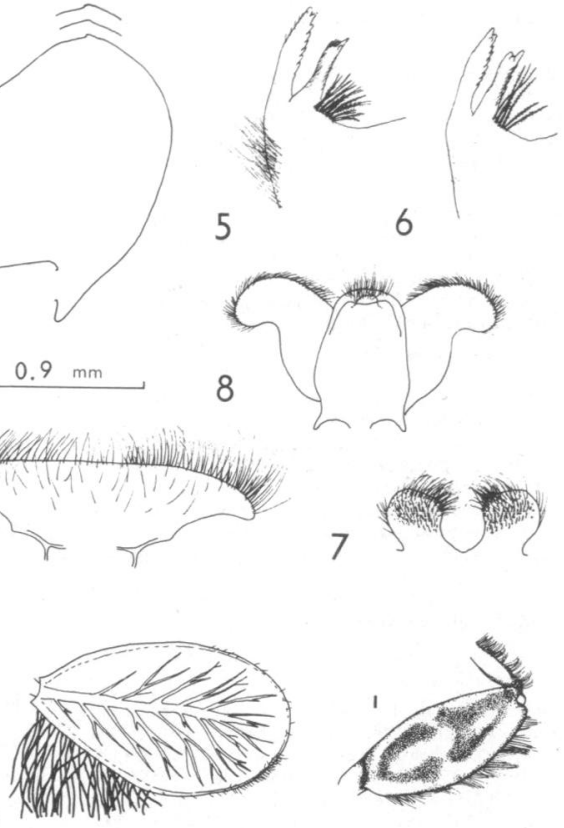

7

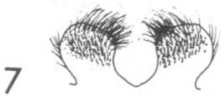

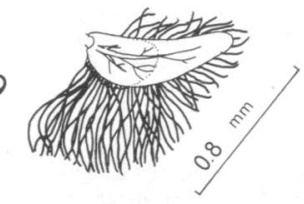

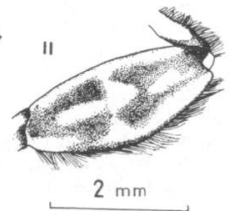

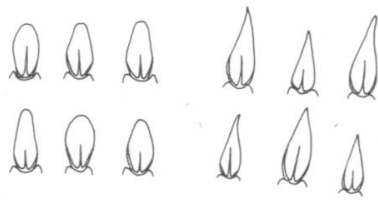

12

13

11

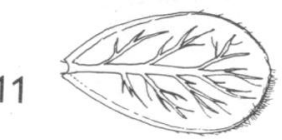

$2.1 \mathrm{~mm}$

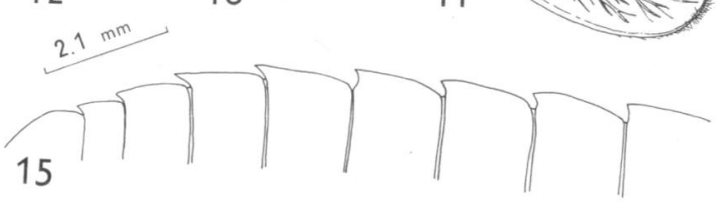

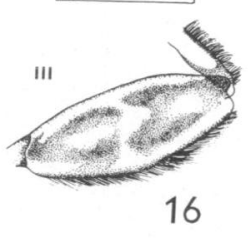

Fig. 1 à 16. - Larves d'Ecdyonurus spp. au dernier stade. Fig. 1,3,4,5, 7-12, 14-16: E. rothschildi Navás. Fig. 2, $13: E$. dispar (Curtis). Fig. $6:$ E. aurantiacus (Burmeister). 1,2: tête, vue dorsale. $3:$ pronotum. $4:$ labre. 5,6: prostheca, $7:$ glosse. $8:$ hypopharynx. $9,10,11: 1^{\mathrm{e}}, 3^{\mathrm{e}}$ et $7^{\mathrm{e}}$ branchies. 12,13 : épines de la surface dorsale des fémurs. $14:$ épines bordantes des tergites. 15 : épines latérales de l'abdomen. 16: coloration de la face dorsale des fémurs droits I. II et III. 


\section{6. - Clé de détermination (larves au dernier stade)}

1. Labium portant des glosses a contour apical en « toit *, en $\propto \mathrm{V}$, renversé. Epines de la surface des fémurs pointues au sommet. Labre étroit (rappor largeur totale/longueur d'insertion : $1,69-2,04$ ). Tergites abdominaux sans paire de bandes latérales obliques brun foncé .....

E. dispar (Curtis).

- Labium portant des glosses à contour apical presque rectiligne et perpendiculaire au plan de symétrie. Epines de la surface des fémurs arrondies au sommet. Labre plus large (rapport largeur totale/longueur d'insertion : $2,26-2,86$ ). Tergites abdominaux avec une paire de bandes latérales obliques brun foncé ....... 2

2. Prostheca composée de 10.15 fortes soies. Tête avec un rapport largeur/longueur de 1,42 -1,55. Pronotum: expansions paranotales reliées au mésothorax et denticule des angles antérieurs absent ou très réduit .

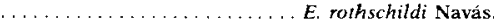

- Prostheca composée de 5-8 fortes soies. Téte plus étroite, avec un rapport largeur/longueur de 1,62 - 1,80. Pronotum : expansions paranotales libres et denticule des angles antérieurs plus long et pointu ............ ................... E. aurantiacus (Burmeister).

\section{7. - Matériel examiné}

\section{E. rothschildi Navás :}

- Algérie, wilaya de Tlemcen, bassins des oueds Isser, Tafna et Sidna Youcha: O. Tafna, 4-22/V/1981, 22-23/XII/1981, 18/TV/1984; O. Isser, 4-22/V/1981; 0 . Chouly, 11.25/XI/1981，22.23/XI1/1981, 16/X/1982, 15/XII/1982, 31/XII/1982, 11/III/1983, 10/IV/1983; O. Bou Hadi, 4-22/V/1981, 15-25/X/1981, 15-25/XI/1981, 23/V//1982, 11/III/1983; O. Sikkak, 21/1/1983; O. Zitoun, 4-22/V/1981, 24/1X.2/X/1981; O. Bou Messaoud, 4-22/ V/1981, 22-23/XII/1981, 27/V/1983; O. Mouilah, $4-22 / \mathrm{V} / 1981$; O. Oua Am, 4.22/V/1981; O. Khemis, 4-22/V/1981, 11-25/XI/1981: O. Sebdou, 4-22/V/1981. 24/IX-2/X/1981; O. Sidna Youcha, 4-22/V/1981; J. Gagneur, 927 larves.

- Algérie, wilaya de Blida, Gorges de la Chiffa, 8-9/IX/1981. Wilaya de Constantine, oued Rhumel et oued Merzoug. 30/IX/1981, T. Soldán, 65 larves.
E. aurantiacus (Burmeister) :

- France : dpt. de la Haute Garonne, la Garonne en arnont de Toulouse, $V$ et VI 1965, A.G.B. Thomas, 28 larves.

- Tchécoslovaquie : Bohême Centrale, la Berounka, PrahaLahovice, 30/X/1977; Bohème du Sud, la Luznice, Bechyne, $21 / \mathrm{VIII} / 1974$; Slovaquie Centrale, le Váh, Králova Lehota, 1/NIII/1976, T. Soldán, 125 larves.

E. dispar (Curtis) :

— France : dpt. de l'Ariège, le Volp à $320 \mathrm{~m}, 13 /$ VIIL/1967, A.G.B. Thomas, 3 larves.

- Tchécoslovaquie : Bohême du Sud, le nuisseau Zidova strouha, 20/VIII/1979; Slovaquie Orientale, le ruisseau Okna, 11/VII/1975, T. Soldán, 42 larves.

\section{Remerciements}

Nous remercions vivement M. A.G.B. Thomas pour le don de son matériel et pour avoir relu et critiqué le manuscrit.

Travaux cltés

Lestage (J.A.) 1925. - Ephéméroptères, Plecoptères et Trichopteres recueillis en Algérie par M.H. Gauthier et liste des espèces connues actuellement de l'Afrique du Nord. Bull. Sac. Hist. nat. Afr. N., $16: 8.18$.

Navás (L.). 1929. - Insectes Névroptères et voisins de Barbarie (Septieme série). Bull. Soc. Hist. nat. Afr, N., 20:57.60.

Puthz (V.) 1978. - Ephemeroptera. In J. Illies : Limnotauna Europae (pp. 250-263). Fischer Verlag, Stuttgart.

Thomas (A.). 1968. - Sur la taxonomie de quelques especes d'Ecdyonurus du Sud-Ouest de la France (Ephemeroptera). Annls Limnol, 4 (1): 51-71.

Thomas (A.G.B.) et Dakki (M.). 1979. - Ephéméroptères d'Afrique du Nord : I. Ecdyonurus rotschildi Navás, 1929. Description des imagos. Annis Limnol., 14 (3) : 197-201. 